\title{
Construcción y valoración psicométrica de un instrumento de percepción de riesgo de problemas ambientales para la ciudad de Córdoba (Argentina)
}

\author{
Maria Argüello Pitt ${ }^{1}$, Anabel Belaus ${ }^{1}$, Cecilia Reyna ${ }^{1}$
}

18 de septiembre, 2021

\section{Resumen}

El objetivo de esta investigación fue construir y validar un instrumento para medir la percepción de riesgo de problemas ambientales en la ciudad de Córdoba, Argentina. El estudio comprendió cinco etapas, a lo largo de las cuales participaron 506 ciudadanos/as. Se condujeron análisis de datos cualitativos (análisis de contenido) y cuantitativos (análisis descriptivos, análisis de la estructura factorial, análisis de consistencia interna). Se construyó un instrumento que comprende ocho problemas ambientales de relevancia local que se evalúan en nueve características. A partir de la evaluación de distintos agrupamientos de ítems es posible utilizar el cuestionario de diferentes maneras: a) escala general de percepción de riesgo de problemas ambientales que comprende todos los problemas y características; b) escala breve de percepción de riesgo global de problemas ambientales; y c) escalas individuales para cada uno de los problemas ambientales. En general, la evidencia de validez y confiabilidad obtenida para cada uno de los agrupamientos de ítems habilitan su uso en la ciudad de Córdoba, pudiendo utilizarse como herramienta para el manejo y gestión de riesgos.

Palabras clave: percepción de riesgo, problemas ambientales, psicometría, Argentina

\footnotetext{
${ }^{1}$ Instituto de Investigaciones Psicológicas (IIPSI), Consejo Nacional de Investigaciones Científicas y Técnicas (CONICET), Facultad de Psicología, Universidad Nacional de Córdoba (UNC), Argentina. Contacto: ceciliareyna@unc.edu.ar
} 


\section{Introducción}

A medida que se intensifica el cambio climático, las ciudades se vuelven más vulnerables a su impacto. Se espera que cerca del $70 \%$ de la población mundial viva en ciudades para el año 2050 y esté expuesta a condiciones extremas si no se toman medidas urgentes para modificar los entornos urbanos. Por consiguiente, las estrategias para la mitigación y para una gestión adaptativa del riesgo ambiental se tornan sumamente necesarias (WMO, UNEP, IPCC, GFCS, 2019).

Estudios realizados en Argentina evidencian la situación crítica en la que se encuentran algunos sectores de la población debido a la exposición a numerosos problemas ambientales. Entre los principales problemas ambientales urbanos se encuentra la falta de sistemas de agua potable para abastecer a toda la población, la inadecuada provisión de sistemas de evacuación de efluentes, la contaminación del agua y la escasez de espacios verdes (Clichevsky, 2002). En la ciudad de Córdoba, la segunda ciudad más poblada de Argentina, entre los problemas ambientales más riesgosos se encuentran la proliferación de basurales a cielo abierto, el vertido de desagües cloacales a la vía pública, la contaminación del aire y la contaminación de la napa freática (Martínez de Rusconi \& Maffrand, 2014; Red Ciudadana Nuestra Córdoba, 2014).

La percepción de riesgo cumple un rol clave en la adopción de conductas de cuidado del medio (Corral Verdugo et al., 2003) y en acciones de mitigación de riesgo frente a problemas ambientales específicos (Martin et al., 2009; Ohe \& Ikeda, 2005). Es más probable que las personas desarrollen conductas de prevención y protección del medio ambiente si perciben y evalúan adecuadamente los riesgos a los que están expuestas y, además, conocen y se les comunica de manera efectiva la forma de poder controlarlos (Martínez Soto, 2004). Diversos investigadores han realizado aportes para la comprensión y medición de la percepción de riesgo de problemas ambientales, con un amplio abanico de definiciones conceptuales e instrumentos de evaluación. Así, a partir de los desarrollos teóricos y empíricos previos, y teniendo en cuenta el carácter contexto-dependiente de los problemas ambientales, nos propusimos avanzar en la construcción y validación de un instrumento para medir la percepción de riesgo de problemas ambientales adaptado a la ciudad de Córdoba, Argentina.

\subsection{Paradigmas en el estudio de la percepción de riesgo}

La literatura científica ha sugerido varias definiciones para la percepción de riesgo, siendo abordado principalmente desde tres paradigmas. Como señala Weber (2001), el modelo 
axiomático propone que las personas transforman la información objetiva acerca del riesgo en términos que reflejan el impacto en sus propias vidas. Por otra parte, el modelo sociocultural se asienta en la idea de que la sociedad modela los intereses de las personas y qué variables y eventos plantean riesgos para esos intereses y formas de vida. Por último, los estudios desde el modelo psicométrico postulan que la percepción de riesgo es afectada tanto por la información objetiva y científica como por otros factores, como las experiencias personales, las actitudes y los sentimientos asociados a un problema o situación riesgosa.

El paradigma psicométrico ha sido el dominante en el estudio de la percepción de riesgo (Rohrmann, 2008; Sjöberg, 1996). Slovic y colaboradores fueron los primeros en sostener que la percepción de riesgo y sus características son constructos psicológicos que pueden ser medidos a través de métodos psicométricos (Sjöberg, 1996). Desde este enfoque se sostiene que cuando las personas evalúan el riesgo realizan una inferencia de la probabilidad de ocurrencia de un fenómeno y una previsión de las consecuencias en base a ciertos factores sociales y psicológicos, como creencias dominantes, opinión pública y decisiones políticas, emociones y sentimientos asociados (Stanojlovic, 2015). La forma en la cual las personas evalúan la magnitud del riesgo de un evento depende, en parte, de ciertos atributos o características del problema, de la persona y de la sociedad. Algunas características son la frecuencia del problema, la inmediatez de las consecuencias, el poder de destrucción del problema, la relación de cercanía de la persona con el problema, y la regulación por parte de las autoridades (Bronfman \& Cifuentes, 2003; Mayer et al., 2017; McDaniels et al., 1995; Rufat, 2015). Las características han mostrado tener un peso singular en la valoración del riesgo según el tipo de experticia. Por ejemplo, en población general tienen mayor peso características como potencial catastrófico del problema, fatalidad de las consecuencias y falta de control; mientras que en expertos/as tienen mayor peso factores basados en evidencia empírica, p.e., el índice de mortalidad anual (Slovic \& Weber, 2002).

\subsection{Medición de la percepción de riesgo ambiental}

Existe una diversidad de instrumentos para medir la percepción de riesgo de problemas ambientales. Algunos instrumentos se focalizan en evaluar la percepción de riesgo de un problema ambiental específico, por ejemplo, incendios (Martin, et al., 2009), consumo de agua (Rodriguez-Sanchez \& Sarabia-Sanchez, 2017), manejo de la basura (Linzalone et al., 2017), entre otros. En cambio, otros instrumentos comprenden una diversidad de problemas riesgosos, llegando incluso a evaluar 65 problemas en un único cuestionario. 
Al mismo tiempo, existen diferencias en cuanto a las características que se emplean para describir la percepción de riesgo. McDaniels et al. (1995), exponentes del paradigma psicométrico clásico, indagaron en un mismo instrumento valoraciones de hasta 31 características para 65 problemas. La desventaja de este tipo de instrumento es la marcada extensión y, además, que algunas características resultan disonantes o no apropiadas para algunos problemas. En el otro extremo, otros instrumentos, por ejemplo el utilizado por Weber et al. (2000), evalúan la percepción de riesgo utilizando sólo una característica global. La desventaja asociada a este tipo de instrumentos es que se pierde especificidad en la descripción de la percepción de riesgo de cada problema ambiental.

En línea con el amplio abanico de instrumentos, se aprecia que la evidencia de las propiedades psicométricas es diversa. Los estudios que han avanzado en la comprensión de la estructura subyacente recurren a análisis exploratorios y/o confirmatorios. En el estudio de McDaniels et al. (1995), conducido con habitantes de Vancouver, se aplicó análisis de componentes principales y se identificaron cinco factores subyacentes: conocimiento de los impactos, impacto en las especies, impacto en los humanos, beneficios asociados y evitación. Weber et al. (2000) observaron, en una muestra de estadounidenses, una estructura unidimensional en un conjunto de 32 problemas ambientales evaluados según la percepción de riesgo general, con adecuada consistencia interna. Marcon et al. (2015), en una muestra de italianos/as, observaron una estructura unidimensional subyacente a siete problemas ambientales evaluados según el riesgo para la salud, con adecuada evidencia de consistencia interna.

En el ámbito local, Mikulic y colaboradores, investigadores/as pioneros/as en el campo de estudio de la percepción de riesgo en Argentina, observaron una estructura bidimensional subyacente a 13 problemas riesgosos (Mikulic, Casullo, Fernández et al., 2012) evaluados según el daño para uno/a mismo/a, el primer factor reunía ítems sobre fuentes de peligro de origen tecnológico-natural y el segundo factor ítems sobre fuentes de peligro de origen humano, con índices de consistencia interna adecuados (Caballero \& Mikulic, 2017). Además, adaptaron el cuestionario de evaluación del riesgo de Rohrmann en una muestra de población general y analizaron las diferencias según género (Mikulic et al., 2009) y también evaluaron el modelo de percepción de riesgo desarrollado por Rohrman en muestras de estudiantes a través de un modelo de ecuaciones estructurales (Mikulic, Casullo, Crespi, et al., 2012). 


\subsection{El presente estudio}

La literatura sobre percepción de riesgo de problemas ambientales no proporciona una imagen unívoca, en términos conceptuales ni instrumentales. Además, vale remarcar que los riesgos ambientales son contexto-dependientes. De allí la importancia de construir un instrumento que tenga en cuenta ítems de problemas considerados como riesgosos por la población meta y que recupere las características más relevantes para describirlos.

Estudios realizados en la ciudad de Córdoba resaltan la situación crítica en la que se encuentran los/as ciudadanos/as debido a la exposición a importantes riesgos ambientales (Martínez de Rusconi \& Maffrand, 2014). Ante ello, decidimos construir un instrumento que, considerando la literatura del área, permita capturar las particularidades locales para medir la percepción de riesgo asociada a problemas ambientales. Se implementó un estudio instrumental que comprendió la construcción del instrumento y la obtención de evidencia de validez y confiabilidad con muestras de adultos/as de la ciudad de Córdoba (Argentina). Se desarrollaron cinco etapas cuyos objetivos, metodología y resultados se describen debajo. Para mayor detalle de cada etapa se puede consultar el anexo.

\section{Aspectos éticos}

A lo largo de toda la investigación se respetaron los lineamientos éticos para la investigación con humanos recomendados por la Asociación Psicológica Americana (APA, 2017), las pautas para la investigación establecidas en códigos de ética locales (CPPC, 2016; FePRA, 2013) y se respetó la Ley Nacional No 25.326 de protección de datos personales. Se utilizó consentimiento informado y se tomaron medidas para garantizar el respeto por los derechos humanos, el cuidado y preservación del medio ambiente y de las generaciones futuras.

\section{Etapa 1}

El objetivo de esta etapa fue describir aspectos formales de instrumentos de medición de percepción de riesgo de problemas ambientales y también identificar en la literatura científica problemas ambientales y características potencialmente relevantes para los/as ciudadanos/as de Córdoba.

\subsection{Método y Resultados}

Se realizó una revisión no sistemática de instrumentos que medían percepción de riesgo de problemas ambientales desde el paradigma psicométrico. Se revisaron 14 estudios, de los cuales se consideró la cantidad de problemas ambientales estudiados, la cantidad de 
características, el formato de respuesta y el rango de la escala de respuesta. Se observó gran diversidad con respecto a la cantidad de problemas (1 a 73), y también respecto a cuáles y cuántas características (1 a 31) de la percepción de riesgo se evalúan (Anexo A, Tabla 1).

Se identificaron 44 ítems sobre problemas ambientales potencialmente relevantes para nuestro contexto. Se descartaron reactivos que no correspondían a las condiciones de la ciudad de Córdoba (p.e., minería a cielo abierto) y reactivos relacionados específicamente con el fenómeno de cambio climático (p.e., reducción de la capa de ozono). Tras la consulta con un/a experto/a en el área ambiental, quedó definido un banco inicial de 22 ítems potencialmente relevantes para los/as ciudadanos/as de Córdoba (Anexo A, Tabla 2).

En cuanto a las características de los problemas ambientales, de las 20 características identificadas en los instrumentos revisados, se seleccionaron 16 que potencialmente podrían ser respondidas para todos los problemas que formaban parte del banco de ítems y que eran mutuamente excluyentes (Anexo A, Tabla 3).

\section{Etapa 2}

Los objetivos en esta etapa fueron recuperar eventos considerados como problemas ambientales riesgosos por parte de habitantes de la ciudad de Córdoba y expertos/as en temas ambientales, identificar las características de los problemas que influyen en tal percepción y diseñar un instrumento para medir la percepción de riesgo de problemas ambientales.

\subsection{Método}

3.1.1. Participantes. Participaron cinco personas de la población general de Córdoba de 23 a 57 años $(M=35.4, D S=14)$, tres mujeres y dos varones, seleccionados/as por conveniencia, y tres expertas en temas ambientales de 42 a 62 años $(M=53.33, D S=10.26)$ seleccionadas de manera intencional.

3.1.2. Procedimiento e instrumentos. Se condujeron entrevistas semi-estructuradas (ver Anexo B, Guía de preguntas). El cuestionario comprendía preguntas abiertas y cerradas referidas a los 22 problemas ambientales y las 16 características seleccionadas en la etapa 1. Primero, las personas puntuaron cada problema ambiental según la percepción de riesgo global $(1=$ nada riesgoso, 5 = muy riesgoso). Segundo, la entrevistadora seleccionó los cinco problemas con mayor puntaje y los/as participantes los ordenaron de mayor a menor riesgo percibido. Tercero, los/as participantes seleccionaron las cinco características que consideraban más representativas para cada uno de los tres problemas. Además, se condujo una entrevista no 
estructurada adicional con una experta en el área ambiental para clarificar la ocurrencia en la ciudad de Córdoba de algunos problemas ambientales que surgieron en las entrevistas. En una instancia final, con el equipo de investigación que integran las autoras, se definió el rango de respuesta y la redacción de las opciones de respuesta de las características seleccionadas.

3.1.3. Análisis de datos. Se realizaron análisis descriptivos y análisis de contenido (López et al., 2010).

\subsection{Resultados}

\subsubsection{Problemas ambientales}

A partir del análisis cuantitativo (Anexo B, Tabla 1) y cualitativo de las respuestas de los/as ciudadanos/as y de los/as expertos/as se seleccionó un conjunto de ítems del banco inicial, lo cual implicó reagrupamiento de ítems y modificación de la redacción (Anexo B, Tabla 2). Además, los/as ciudadanos/as propusieron problemas adicionales, cuatro (Anexo B, Tabla 3) de los cuales fueron incorporaron al conjunto de 13 ítems de la primera versión del instrumento (Anexo B, Tabla 4) tras la consulta con la experta.

\subsubsection{Características}

A partir del análisis cuantitativo (Anexo B, Tabla 5) y cualitativo de las respuestas de los/as entrevistados/as y del juicio de la/el experta/o ambiental se definieron las características a incluir en la primera versión del instrumento. Se agruparon ítems semejantes y se excluyó el ítem "control por parte de la población" dado que generaba dudas al responder. Se incluyó la característica "cantidad de personas afectadas" y "riesgo global". Se definió una escala de respuesta de cinco puntos. La primera versión quedó conformada por 10 características (Anexo B, Tabla 6).

\section{Etapa 3}

Los objetivos de esta etapa fueron los mismos que en la Etapa 2 pero se empleó una muestra mayor de ciudadanos/as de Córdoba.

\subsection{Método}

4.1.1. Participantes. Participaron 58 residentes de la ciudad de Córdoba de 20 a 39 años (36 personas brindaron información sobre edad, $M=28.28$ años, $D S=5.61$; y sexo, 26 mujeres y 10 varones). Se empleó muestreo autoelegido. De las 58 personas, 5 sólo respondieron una 
pregunta inicial sobre qué implicaba para ellos/as los riesgos ambientales, en tanto que 53 respondieron al menos sobre un problema ambiental.

4.1.2. Instrumentos. Se utilizó una encuesta online que comprendió tres apartados: a) preguntas abiertas sobre cambio climático y problemas ambientales (no analizado en este estudio); b) preguntas sobre los 13 problemas ambientales definidos en la primera versión preliminar del instrumento, cada uno con diez características; y c) información socio-demográfica (Anexo C, Cuestionario).

4.1.3. Procedimiento. La invitación se realizó a través de redes sociales. Las personas completaron la encuesta en LimeSurvey, tras recibir información general del estudio y brindar su consentimiento (Anexo C, Consentimiento). En un primer período de recolección (8 días) se obtuvieron 26 respuestas, por lo que se decidió incorporar premios para incentivar la participación en un segundo período (18 días).

4.1.4. Análisis de datos. Se consideraron los casos que respondieron a todas las características de uno o más problemas. Se realizaron análisis cuantitativos descriptivos. Para cada problema se consideró el "riesgo global" y, por otra parte, las demás características. Para evaluar las características se consideraron, en un primer momento, todos los problemas y, luego, los que fueron evaluados como más relevantes en esta etapa. Se siguió un proceso iterativo de análisis de problemas y características.

\subsection{Resultados}

\subsubsection{Problemas ambientales}

Once de los 13 problemas recibieron puntajes altos en riesgo global (media superior a 4), de los cuales nueve también puntuaron alto en las demás características (media superior a 4) (Anexo C, Tabla 1). Luego de analizar las características, se seleccionaron ocho problemas para la segunda versión del cuestionario: basurales, desborde de cloacas, pesticidas, deforestación, contaminación industrial, incendios, derroche de agua, contaminación vehicular.

\subsubsection{Características}

La característica de los distintos problemas ambientales con la media más alta fue "duración de las consecuencias", seguida por "impacto sobre la salud", mientras que "cercanía al espacio cotidiano" presentó la media más baja (Anexo C, Tabla 2). Semejantes resultados se observaron al analizar las características de los ocho problemas seleccionados en esta etapa (Anexo C, Tabla 3). En cada problema se identificaron las cinco características con mayor promedio y, luego, la cantidad de veces que cada característica había quedado dentro del top- 
5 para los distintos problemas. "Impacto sobre la salud" estuvo presente en el top-5 en siete de ocho problemas, mientras que "cercanía al espacio cotidiano" sólo en un problema. En base a estos resultados se decidió excluir esta característica en etapas siguientes y considerar ocho características específicas de problemas ambientales, a las cuales se añade la percepción de riesgo global.

\section{Etapa 4}

El objetivo de esta etapa fue evaluar el contenido y la comprensión de la versión preliminar del instrumento de percepción de riesgo de problemas ambientales de la ciudad de Córdoba. Se condujo un estudio con expertos/as y otro con población general.

\section{A. Estudio con expertos/as}

\subsection{Método}

5.1.1. Participantes. Participaron cuatro expertos/as en temas ambientales de la ciudad de Córdoba de 34 a 61 años $(M=52, D S=12.35)$, dos mujeres y dos varones, de distintas áreas de especialización, seleccionados/as de manera intencional.

5.1.2. Instrumentos y procedimiento. Las entrevistas se condujeron de manera presencial y fueron grabadas. Las personas recibieron información sobre el estudio y luego brindaron su consentimiento. Se utilizó un cuestionario semi-estructurado que comprendió los siguientes apartados: a) datos sociodemográficos y experiencia en temas ambientales; b) actividad sobre problemas ambientales; y c) actividad sobre las características de problemas ambientales (Anexo D, Guía de preguntas).

5.1.3. Análisis de datos. Se realizaron análisis descriptivos cuantitativos y análisis de contenido siguiendo la propuesta de López et al. (2010).

\subsection{Resultados}

\subsubsection{Problemas ambientales}

Los/as expertos/as ordenaron los problemas ambientales según el riesgo asociado en el marco de la ciudad de Córdoba (Anexo D, Tabla 1). El ítem "incendios" fue ubicado en último lugar (menor riesgo) por todos/as y se decidió excluirlo. Además, mencionaron varios problemas ambientales que no incluía la segunda versión del cuestionario. El común denominador fue la referencia a la "expansión urbana territorial" (con expresiones como "mal 
ordenamiento territorial”, "planificación de la ciudad”, "expansión urbana sobre la producción hortícola", "expansión de la localización de áreas urbanas" y "uso inadecuado del suelo") y las "inundaciones" (con expresiones como "el impacto hídrico de algunas problemáticas es importante por las inundaciones (...) las cuencas en la ciudad no van a ser suficientes y se va a inundar cada vez más"), problemas que fueron incluidos en la tercera versión del cuestionario.

Por sugerencia de los/as expertos/as se realizaron cambios en la redacción de cuatro problemas, que quedaron de la siguiente manera: "mal tratamiento de efluentes: desborde de cloacas, falta de cloacas y/o aumento de napas", "mala gestión de residuos y/o basurales", "falta de forestación", "problemas con la red de agua: contaminación y/o escasez".

\subsubsection{Características}

Los/as expertos/as indicaron qué características consideraban como más adecuadas para describir los problemas ambientales como riesgosos. Las respuestas fueron codificadas en una escala de menor (1) a mayor adecuación (3). La característica "impacto sobre la pérdida de especies animales y/o vegetales" obtuvo el menor puntaje (Anexo D, Tabla 2). Incluso, los/as expertos/as sugirieron que esa característica resultaba confusa para algunos ítems, por lo que se decidió excluirla.

\section{B. Estudio con población general}

\subsection{Método}

6.1.1. Participantes. Participaron cinco ciudadanas/os de 26 a 65 años $(M=43.40, D S=18.65)$, tres mujeres y dos varones, con distintos niveles educativos, seleccionadas/os por conveniencia.

6.1.2. Instrumento. Se administró la tercera versión del cuestionario de percepción de riesgo de problemas ambientales, en conjunto con una serie de preguntas para evaluar el contenido y la comprensión del cuestionario (Anexo F, Guía de preguntas).

6.1.3. Procedimiento. Se condujeron entrevistas presenciales. Inicialmente las personas brindaron su consentimiento tras recibir información del estudio. Luego, completaron el cuestionario mientras se aplicaba la técnica de sondeo verbal de comprensión y de parafraseo (Caicedo Cavagnis \& Zalazar-Jaime, 2018). Las entrevistas fueron grabadas. Después del análisis de datos se consultaron algunos aspectos con el equipo de investigación para definir la versión final del cuestionario.

6.1.4. Análisis de datos. Se siguieron los pasos sugeridos por Caicedo Cavagnis y ZalazarJaime (2018). 


\subsection{Resultados}

\subsubsection{Problemas ambientales}

A partir del análisis realizado, se revisó la redacción de algunos ítems. El problema "mala gestión de residuos" era asociado sólo a la recolección de residuos, aunque también se refiere a la contaminación por basura, por lo que se redactó como "mala gestión de residuos y/o basurales". También hubo dificultades para comprender la palabra "napas", por lo que el ítem se redactó como "mal tratamiento de efluentes: desborde y/o falta de cloacas". Además, en los problemas "uso de pesticidas" e "inundaciones" algunos/as participantes respondieron en base a la Provincia, no a la Ciudad, por lo cual se incluyó la siguiente aclaración "Recordá que estamos pensando en la ciudad de Córdoba”. Por último, los/as participantes mostraron notorias dificultades para comprender el problema "expansión territorial urbana". Tras la revisión con el equipo de investigación, se decidió descartar este problema ambiental.

\subsubsection{Características}

Algunos/as participantes expresaron dudas sobre la comprensión de la característica “predictibilidad” (p.e., “¿Significa qué tanto se lo ve?”, “¿se refiere a qué tan predecible es que ocurra el problema o qué tan predecible es que se solucione?”, “esta fue la que más me costó”), por lo que se decidió no incluirla en la versión final del instrumento. Por otra parte, se observó que las personas hacían referencia a una característica ampliamente utilizada en la literatura, "posibilidad de control de la población" (p.e., "las personas no clasifican correctamente los residuos", "nosotros también contaminamos las calles"), por lo que se la incluyó en la versión final del cuestionario a pesar de que se la había excluido en la etapa 2. Los/as participantes también hicieron referencia al control gubernamental (p.e., "no hay una buena organización del gobierno", "el sistema de recolección no funciona como debería funcionar"), por lo que se incluyó la característica “posibilidad de regulación gubernamental".

\section{Etapa 5}

El objetivo de esta etapa fue obtener evidencia de consistencia interna y validez estructural del Cuestionario de Percepción de Riesgo de Problemas Ambientales en una muestra de adultos/as de la ciudad de Córdoba (Argentina).

\subsection{Método}

7.1.1. Participantes. Participaron 413 residentes de la ciudad de Córdoba de 18 a 70 años (M $=32.4, D S=11.3), 251(60.8 \%)$ mujeres, $161(39 \%)$, hombres y $1(0.2 \%)$ persona prefirió no 
indicar su sexo. El nivel educativo fue el siguiente: $2.2 \%$ secundario incompleto o menos, $9.7 \%$ secundario completo, $5.1 \%$ terciario incompleto, $10.7 \%$ terciario completo, $41.4 \%$ universitario incompleto, $22 \%$ universitario completo, $5.3 \%$ postgrado incompleto y $3.6 \%$ postgrado completo. El tipo de muestreo fue autoelegido.

7.1.2. Instrumento. Se utilizó la versión final del Cuestionario de Percepción de Riesgo de Problemas Ambientales construido en las etapas precedentes, que comprende ocho problemas ambientales que se evalúan en nueve características (Anexo G, Cuestionario). El formato de respuesta es tipo Likert de cinco puntos. También se realizaron preguntas sobre percepción de riesgo de cambio climático que forman parte de un estudio mayor y no fueron analizadas para esta investigación. Se recabó información sobre sexo, edad y nivel educativo. Finalmente, se requirió de manera opcional información de contacto para participar en un sorteo por premios. 7.1.3. Procedimiento. Las personas fueron invitadas a través de redes sociales. El cuestionario se aplicó a través de Google Forms. Las personas brindaron su consentimiento informado (Anexo G, Consentimiento) y luego respondieron el cuestionario.

7.1.4. Análisis de datos. Primero, se condujeron análisis cuantitativos descriptivos de las características para cada uno de los problemas ambientales. Segundo, se evaluó la estructura factorial. Se condujeron los siguientes análisis: 1) análisis factorial exploratorio (AFE) del conjunto total de ítems (Escala general); 2) análisis factorial confirmatorio (AFC) de los ítems de percepción de riesgo global de los distintos problemas ambientales, bajo el supuesto de una dimensión subyacente (Escala breve); y 3) AFC para cada uno de los problemas ambientales, bajo el supuesto de dos dimensiones subyacentes en cada problema (a partir de lo observado en el AFE, Escala de cada problema). Para el AFE se empleó rotación geomin para facilitar la interpretación de los resultados. Tanto en el AFE como en el AFC las variables fueron tratadas como categóricas y se empleó el método de estimación de mínimos cuadrados ponderados con media y varianza ajustada (WLSMV por sus siglas en inglés). Se consideraron los siguientes índices de bondad de ajuste: $\chi 2$, residuo cuadrático medio estandarizado (SRMR, .05-.08, sólo para AFE), error cuadrático medio de aproximación (RMSEA,.05-.08, IC 90\%), índice de Tucker-Lewis (TLI, >.95) e índice de ajuste comparativo (CFI, >.95) (Hu \& Bentler, 1999; Lloret et al., 2017). Para interpretar las cargas factoriales, se consideró el valor de .40 (o superior) como indicativo de representación del factor (Tabachnick \& Fidell, 2013). Finalmente, se estimó para cada modelo el coeficiente Alfa de Cronbach y el coeficiente Omega de McDonald. 
Se emplearon los programas Mplus 6 (Muthén \& Muthén, 1998-2011), R 3.5.1 (R Core Team, 2018) .y el paquete MBESS (v4.8, Kelley, 2007).

\subsection{Resultados}

\subsubsection{Análisis descriptivos}

La característica "posibilidad de regulación del gobierno" obtuvo el mayor puntaje medio en 7/8 problemas ambientales, mientras que "posibilidad de control de la población expuesta" obtuvo el menor puntaje medio en 7/8 problemas. La característica "posibilidad de control de la población expuesta" presentó las desviaciones estándar más altas (Anexo G, Tabla 1).

7.2.2. Estructura y consistencia interna: Percepción de riesgo de todos los problemas ambientales en conjunto (Escala general)

La Tabla 1 muestra los índices de ajuste global de todos los modelos evaluados. En el AFE se evaluaron modelos de 1 a 5 factores. El modelo de cuatro factores mostró un ajuste aceptable en gran parte de los indicadores. En la Tabla 2 del Anexo G se muestran las cargas factoriales. El primer factor se denominó "problemas ambientales urbanos", incluye tres problemas: contaminación vehicular, mala gestión de residuos y/o basurales, y mal tratamiento de efluentes: desborde de cloacas y/o falta de cloacas, cada uno asociado a las mismas seis características: riesgo global, cantidad de personas afectadas, extensión del área afectada, frecuencia, impacto en la salud y duración de las consecuencias. El segundo factor se denominó "problemas ambientales de la periferia", incluye tres problemas: contaminación industrial, falta de forestación, y uso de pesticidas, cada uno asociado a las mismas características que los problemas del primer factor. El tercer factor se denominó "posibilidad de agencia”, incluye las siguientes tres características de los ocho problemas ambientales: posibilidad de evitarlo, posibilidad de control de la población, y posibilidad de regulación del gobierno. El cuarto factor se denominó "problemas con el recurso hídrico", incluye dos problemas: inundaciones y contaminación, derroche y/o problemas con la disponibilidad de agua potable, cada uno asociado a las mismas seis características que el primer y segundo factor. Las cargas factoriales de 68/72 ítems presentaron valores superiores a .4. Los cuatro ítems restantes mostraron valores entre .3 y .4 .

Los cuatro factores presentaron valores aceptables de consistencia interna (IC 95\%): factor 1: $\alpha=.88$ (.87-.90), $\omega=.88$ (.82-.94); factor 2: $\alpha=.91$ (.90-.93), $\omega=.91$ (.90-.93); factor 3: $\alpha=.87$ (.85-.89), $\omega=.87$ (.85-.89); factor 4: $\alpha=.87$ (.84-.89), $\omega=.86(.82-.89)$. 
7.2.2. Estructura y consistencia interna: Percepción de riesgo global de problemas ambientales (Escala breve)

El ajuste global del modelo fue aceptable según todos los indicadores considerados (Tabla 1). Las cargas factoriales de los ítems fueron superiores a .4, a excepción del ítem referido al problema contaminación vehicular (Tabla 2). Los valores de consistencia interna (IC 95\%) fueron los siguientes: $\alpha=.69$ (64-.73), $\omega=.69$ (.64-.74).

7.2.3. Estructura y consistencia interna: Percepción de riesgo de cada uno de los problemas ambientales (Escala de cada problema)

El ajuste global para cada uno de los problemas ambientales fue aceptable (Tabla 1). La inspección de las cargas factoriales evidenció, en los distintos problemas ambientales (Tabla 3), el siguiente agrupamiento: el Factor 1 agrupa seis características generales: riesgo global, cantidad de personas afectadas, extensión del área afectada, frecuencia, impacto en la salud y duración de las consecuencias; y el Factor 2 agrupa tres características referidas a la posibilidad de agencia: posibilidad de evitarlo, posibilidad de control de la población expuesta y posibilidad de regulación del gobierno. En general, las cargas factoriales fueron superiores a .4. Las correlaciones entre los factores estuvieron en el rango .325-.677, en todos los casos fueron estadísticamente significativas.

Con respecto a la consistencia interna, para el Factor 1 los valores Alfa de Cronbach fueron de .838 a .910 y los valores Omega de McDonald de .841 a .919; y en el Factor 2 los valores Alfa de Cronbach fueron de .286 a .741 y los valores Omega de McDonald de .465 a .749. Vale señalar que el Factor 2 en cada una de las escalas de problemas ambientales quedó conformado por sólo tres ítems.

Los valores de consistencia interna (IC 95\%) fueron los siguientes: $\alpha=.69$ (64-.73), $\omega$ $=.69(.64-.74)$. 
Tabla 1

Índices de bondad de ajuste de distintos modelos del Cuestionario de Percepción de Riesgo de Problemas Ambientales

\begin{tabular}{|c|c|c|c|c|c|c|c|}
\hline Modelo & $\begin{array}{l}\text { Cantidad } \\
\text { de } \\
\text { variables }\end{array}$ & $X^{2}$ & $g l$ & RMSEA (IC 90\%) & TLI & CFI & $\begin{array}{l}\text { SRM } \\
\text { S }\end{array}$ \\
\hline 1. Problemas y características (AFE) & 72 & $7358.05^{* * *}$ & 2.274 & $.074(0.072-.075)$ & .768 & .793 & .11 \\
\hline 2. Riesgo global, todos los problemas (AFC) & 8 & $30.690 *$ & 2 & $0.036(0.000-0.060)$ & .976 & .983 & \\
\hline 3. Contaminación industrial (AFC) & 9 & $59.255^{* * *}$ & 2.3 & $.062(.043-.081)$ & .988 & .993 & \\
\hline 4. Mala gestión de residuos y/o basurales (AFC) & 9 & $93.546 * * *$ & 2.6 & $.079(.062-.097)$ & .964 & .974 & \\
\hline 5. Falta de forestación (AFC) & 9 & $37.177 * *$ & 2.1 & $.043(.019-.066)$ & .993 & .996 & \\
\hline 6. Contaminación vehicular (AFC) & 9 & $65.274 * * *$ & 2 & $.074(.054-.094)$ & .983 & .99 & \\
\hline $\begin{array}{l}\text { 7. Contaminación, derroche y/o problemas de } \\
\text { disponibilidad de agua potable (AFC) }\end{array}$ & 9 & $43.809 * * *$ & 1.9 & $.056(.034-.078)$ & .989 & .994 & \\
\hline 8. Inundaciones (AFC) & 9 & $52.278^{* * *}$ & 1.8 & $.068(.047-.090)$ & .989 & .994 & \\
\hline 9. Uso de pesticidas (AFC) & 9 & $68.727 * * *$ & 1.7 & $.086(.065-.107)$ & .989 & .995 & \\
\hline $\begin{array}{l}\text { 10. Mal tratamiento de efluentes: desbord } \\
\text { cloacas y/o falta de cloacas (AFC) }\end{array}$ & 9 & 28.467 & 2 & $.032(.000-.057)$ & .997 & .998 & \\
\hline
\end{tabular}

Nota. gl= grados de libertad; RMSEA = error cuadrático medio de aproximación; CI = intervalo de confianza; CFI = índice de ajuste comparativo; TLI = índice de TuckerLewis; SRMR = residuo cuadrático medio estandarizado; AFE = análisis factorial exploratorio; AFC = análisis factorial confirmatorio.

$* p \leq .05, * * p \leq .01, * * * p \leq .001$. 
Tabla 2

Estructura factorial de la percepción de riesgo global de problemas ambientales

\begin{tabular}{ll}
\hline Problema & Factor 1 \\
\hline Contaminación industrial & $.611 * * *$ \\
Mala gestión de residuos y/o basurales & $.550^{* * *}$ \\
Falta de forestación & $.582^{* * *}$ \\
Contaminación vehicular & $.367 * * *$ \\
Contaminación, derroche y/o problemas de disponibilidad de agua & \\
potable & $.547 * * *$ \\
Inundaciones & $.407 * * *$ \\
Uso de pesticidas & $.708 * * *$ \\
Mal tratamiento de efluentes: desborde de cloacas y/o falta de cloacas & $.439 * *$ \\
\hline$* * p \leq .001$ &
\end{tabular}

Tabla 3-ver_doc_aparte-310palabras_añadir_cuando_se_pase_a docx. 
Estructura factorial y consistencia interna de cada uno de los problemas del Cuestionario de Percepción de Riesgo de Problemas Ambientales

\begin{tabular}{|c|c|c|c|c|c|c|c|c|c|c|c|c|c|c|c|c|}
\hline \multirow{3}{*}{ Características } & \multicolumn{16}{|c|}{ Problemas ambientales } \\
\hline & \multicolumn{2}{|c|}{$\mathrm{CI}$} & \multicolumn{2}{|c|}{ MGR } & \multicolumn{2}{|c|}{ FF } & \multicolumn{2}{|c|}{$\mathrm{CV}$} & \multicolumn{2}{|c|}{$\mathrm{CA}$} & \multicolumn{2}{|c|}{$\mathrm{I}$} & \multicolumn{2}{|c|}{ UP } & \multicolumn{2}{|c|}{ MTE } \\
\hline & F1 & F2 & $\mathrm{F} 1$ & $\mathrm{~F} 2$ & $\mathrm{~F} 1$ & F2 & F1 & $\mathrm{F} 2$ & F1 & $\mathrm{F} 2$ & F1 & F2 & F1 & $\mathrm{F} 2$ & F1 & $\mathrm{F} 2$ \\
\hline Riesgo global & .906 & & .799 & & .856 & & .880 & & .900 & & .874 & & .902 & & .807 & \\
\hline Cantidad de personas afectadas & .865 & & .860 & & .896 & & .832 & & .814 & & .838 & & .905 & & .866 & \\
\hline Extensión del área afectada & .828 & & .809 & & .850 & & .749 & & .762 & & .801 & & .828 & & .847 & \\
\hline Frecuencia & .824 & & .765 & & .824 & & .785 & & .861 & & .818 & & .868 & & .851 & \\
\hline Impacto en la salud & .752 & & .708 & & .737 & & .879 & & .565 & & .696 & & .851 & & .759 & \\
\hline Duración de las consecuencias & .640 & & .712 & & .641 & & .742 & & .647 & & 649 & & .834 & & .736 & \\
\hline Posibilidad de evitarlo & & $.311^{\mathrm{a}}$ & & 679 & & .900 & & .868 & & .790 & & .712 & & .761 & & .802 \\
\hline Posibilidad de control de la población afectada & & .454 & & .461 & & .416 & & .730 & & .661 & & .607 & & $.134^{\mathrm{b}}$ & & .299 \\
\hline Posibilidad de regulación del gobierno & & $.294^{\mathrm{a}}$ & & .844 & & .742 & & .666 & & .737 & & .662 & & .811 & & .772 \\
\hline Correlación entre factores & & & & & & & & & & & & & & 77 & .5 & \\
\hline Consistencia interna & & & & & & & & & & & & & & & & \\
\hline Alfa de Cronbach & .891 & .370 & .838 & .492 & .880 & .530 & .882 & .741 & .872 & .564 & .886 & .643 & .910 & .365 & .890 & .286 \\
\hline Omega de McDonald & .894 & .465 & .841 & .524 & .883 & .567 & .882 & .749 & .875 & .606 & .884 & .646 & .919 & & .891 & \\
\hline
\end{tabular}

Nota. CI = contaminación industrial, $\mathrm{MGRB}=$ mala gestión de residuos y/o basurales, $\mathrm{FF}=$ falta de forestación, $\mathrm{CV}=$ contaminación vehicular, $\mathrm{CA}=$ contaminación, derroche y/o problemas con la disponibilidad de agua potable, $\mathrm{I}=$ inundaciones, UP = uso de pesticidas, MTE = mal tratamiento de efluentes: desborde de cloacas y/o falta de cloacas. $\mathrm{F} 1$ = características generales de la percepción de riesgo, $\mathrm{F} 2$ = posibilidad de agencia.

Cargas factoriales y relaciones significativas a un nivel $p<.001$, excepción a que resultó significativo a un nivel $p<.01 \mathrm{y}{ }^{\mathrm{b}}$ que resultó no significativo.

${ }^{\mathrm{c}}=$ en dos problemas ambientales no fue posible computar el coeficiente Omega de McDonald por problemas de convergencia. 


\section{Discusión}

En este estudio nos propusimos construir y obtener evidencia de las propiedades psicométricas de un instrumento para medir la percepción de riesgo de problemas ambientales de la ciudad de Córdoba (Argentina), y logramos un cuestionario que puede ser empleado tanto en contextos de investigación como aplicados.

En estudios iniciales se observó una gran variabilidad de los instrumentos existentes en cuanto a la cantidad y tipo de problemas y características, con una notoria escasez de instrumentos adecuados para el contexto local. Ante ello, decidimos construir un instrumento que responda a la percepción de los problemas ambientales por parte de los/as ciudadanos/as de Córdoba. Luego de varias etapas, seleccionamos ocho problemas ambientales percibidos como riesgosos en la Ciudad de Córdoba: inundaciones; contaminación, derroche y/o problemas con la disponibilidad de agua potable; mala gestión de residuos y/o basurales; mal tratamiento de efluentes, desborde y/o falta de cloacas; contaminación vehicular; contaminación industrial; falta de forestación y uso de pesticidas. Los problemas son valorados en nueve características: cantidad de personas afectadas, extensión del área afectada, frecuencia, impacto en la salud, duración de las consecuencias, riesgo global, posibilidad de evitarlo, posibilidad de control de la población expuesta y posibilidad de regulación del gobierno.

Los hallazgos de este estudio contribuyen a la conceptualización de la percepción de riesgo. La evidencia muestra que la percepción de riesgo global no necesariamente se explica a partir de la sumatoria de las características, y que no toda característica es relevante para todos los problemas, aspecto que debería ser considerado al construir instrumentos para medir la percepción de riesgo, se refiera a o no a temas ambientales.

Un resultado llamativo fue el bajo nivel percibido de posibilidad de control de los problemas ambientales por parte de la población, en contraposición con la elevada percepción de la posibilidad de gestión por parte del gobierno. Este aspecto subraya la necesidad de tener en cuenta la participación de la población en la evaluación de los problemas catalogados como riesgosos y en las decisiones que se adoptan para abordarlos.

La evidencia de las propiedades psicométricas se obtuvo para tres agrupamientos singulares de problemas/características. En primer lugar, se consideraron todos los ítems del Cuestionario General de Percepción de Riesgo de Problemas Ambientales. La estructura interna evidenció un agrupamiento de cuatro factores: problemas ambientales urbanos (situaciones vivenciadas cotidianamente por los ciudadanos/as como la contaminación 
vehicular, valoradas en características generales como la cantidad de personas afectadas), problemas ambientales de la periferia (situaciones que se percibe que ocurren en zonas periféricas de la ciudad, como contaminación industrial, valoradas en características generales), posibilidad de agencia (abarcó los ocho problemas con respecto a la posibilidad de agencia propia o externa), y problemas con el recurso hídrico (p.e., inundaciones, problema valorado con características generales). Dado que algunos ítems mostraron cargas factoriales por debajo de .40 se sugiere que futuros estudios consideren su inclusión o no. Los valores de consistencia interna fueron adecuados $(\alpha=.87-.91, \omega=.87-.91)$. En relación a los extensos instrumentos revisados (p.e., Bronfman \& Cifuentes, 2003; McDaniels et al., 1995), el cuestionario construido resulta abarcativo de los problemas ambientales de la ciudad de Córdoba y, a su vez, recupera las características más relevantes de cada uno. Esto permite disponer de un instrumento de fácil aplicación, cuyo tiempo de respuesta puede variar entre 15 y 20 minutos. Estudios futuros podrían avanzar en la obtención de evidencia confirmatoria de la estructura factorial explorada.

En segundo lugar, se consideró la percepción de riesgo global de los ocho problemas ambientales de la ciudad de Córdoba. Los resultados evidenciaron un ajuste global adecuado. Las cargas factoriales fueron superiores a .40, menos el ítem referido a contaminación vehicular que presentó un valor de .367 . La evidencia de consistencia interna fue adecuada. Esta escala ofrece una alternativa breve para medir la percepción de riesgo global asociada a distintos problemas ambientales. Es un instrumento de rápida y fácil administración. La desventaja es que se reduce la precisión en la medición de la percepción de riesgo.

En tercer lugar, se evaluó cada uno de los problemas ambientales por separado con sus características asociadas. En cada problema se apreciaron dos factores, uno referido a características generales ( 6 ítems) que incluía aspectos tales como riesgo global y cantidad de personas afectadas, y otro referido a la posibilidad de agencia (3 ítems), conformado por características como posibilidad de control de la población expuesta. En general, las cargas factoriales fueron superiores a .40, excepto en algunas características de las siguientes escalas: uso de pesticidas, contaminación industrial y mal tratamiento de efluentes: desborde de cloacas y/o falta de cloacas. La relación entre los factores fue significativa y estuvo entre .325 y .677. Con respecto a la consistencia interna, se obtuvieron valores aceptables para el Factor 1, pero no así para el Factor 2 de algunos problemas. Futuros estudios podrían considerar la inclusión de otras características en este factor como una vía para incrementar la confiabilidad. Los resultados muestran que es posible medir cada uno de los problemas ambientales de manera 
independiente abarcando distintas características para evaluar la percepción de riesgo. Las escalas resultan breves y son fáciles de administrar, lo cual resulta positivo si se pretende medir este constructo junto con otras variables. Se debe tener precaución al emplear escalas de problemas cuya evidencia de consistencia interna del Factor 2 no es adecuada.

Aunque la contribución de esta investigación al campo de estudio de la percepción de riesgos ambientales en general y en nuestro contexto en particular es innegable, no está exenta de limitaciones. El cuestionario construido se centra en la percepción actual del riesgo de distintos problemas ambientales. No obstante, la naturaleza de los problemas ambientales es cambiante y, por ende, la percepción de riesgo es una variable no sólo contexto-dependiente sino también tiempo-dependiente. Por ello, este instrumento resulta útil para el momento actual, en tanto que para usos futuros deberá evaluarse si hay problemas "nuevos" que sea necesario incluir y características que sean más representativas del riesgo que implican.

Además, este instrumento se focaliza en la perspectiva de la población general de la ciudad de Córdoba sobre los problemas ambientales. Nuevos estudios podrían evaluar la percepción de riesgo de grupos específicos y en contextos particularmente expuestos a problemas ambientales. En las primeras etapas de esta investigación incluimos la "cercanía del problema al espacio cotidiano", pero esta característica no formó parte del cuestionario final. Autores como Rufat (2015) consideran que el barrio del/la encuestado/a, pero no la distancia a zonas peligrosas, es un buen predictor de las diferencias en la percepción de riesgo. Así, en futuros estudios sería de interés indagar acerca de la zona de residencia de los/as participantes y disponer de un mapeo espacial de los problemas ambientales.

Otra de las limitaciones se refiere a la no consideración de variables emocionales para la valoración de la percepción de riesgos ambientales. En los últimos años, ha tomado un nuevo impulso la inclusión de factores emocionales en el proceso de evaluación y experimentación del riesgo (ver p.e., Slovic \& Slovic, 2015). La inclusión de este tipo de variables aportaría mayor claridad sobre los componentes de la percepción de riesgo de los problemas ambientales.

\section{Conclusiones}

El instrumento construido resulta una contribución sustancial para la medición de la percepción de riesgo de problemas ambientales en el ámbito local, con importantes implicancias aplicadas, y también regional e internacional, dado que el proceso de construcción seguido se puede convertir en un modelo para lograr instrumentos que abarquen los problemas más relevantes de cada sitio, con sus características distintivas, y sea de fácil y rápida aplicación. 
En función de los resultados, es posible utilizar el Cuestionario de Percepción de Riesgo de Problemas Ambientales en distintos formatos, cada uno con un agrupamiento de ítems particular. Primero, una Escala General que evalúa cuatro factores: problemas ambientales urbanos, problemas ambientales de la periferia, posibilidad de agencia y problemas con el recurso hídrico. Segundo, una Escala Breve de Percepción de Riesgo Global. Tercero, ocho escalas de problemas ambientales que evalúan características generales de la percepción de riesgo y la percepción de la posibilidad de agencia. La evidencia de validez y confiabilidad obtenida habilitan el uso del instrumento en el contexto local, pudiéndose utilizar como herramienta para la gestión de riesgos.

\section{Referencias}

American Psychological Association. (2017). Ethical Principles of Psychologists and Code of Conduct. http://www.apa.org/ethics/code/ethics-code-2017.pdf

Bronfman, N. C., \& Cifuentes. L. A. (2003). Risk perception in a developing country: The case of Chile. Risk Analysis, 23(6), 1271-1285. https://doi.org/10.1111/j.02724332.2003.00400.X

Caballero, R. Y., \& Mikulic, I. M. (2017). Validación de un listado de situaciones de riesgo en Buenos Aires. Anuario de Investigaciones, 24, 269-276. https://www.redalyc.org/pdf/3691/369155966014.pdf

Caicedo Cavagnis, E., \& Zalazar-Jaime, M. F. (2018). Entrevistas cognitivas: Revisión, directrices de uso y aplicación en investigaciones psicológicas. Avaliçãao Psicológica, 17(3), 362-370. https://doi.org/10.15689/ap.2018.1703.14883.09

Clichevsky, N. (2002). Pobreza y políticas urbano-ambientales en Argentina. CEPAL. Serie Medio ambiente y desarrollo. Santiago de Chile: Naciones Unidas.https://repositorio.cepal.org/bitstream/handle/11362/5748/1/S024234_es.pdf

Colegio de Psicólogos de la Provincia de Córdoba (CPPC) (2016). Nuevo código de Ética. https://cppc.org.ar/nuevo-codigo-de-etica-del-colegio-de-psicologos-de-la-provinciade-cordoba/

Corral Verdugo, V., Frías Armenta. M., \& González Lomelí. D. (2003). Percepción de riesgos. conducta proambiental y variables demográficas en una comunidad de Sonora. México. $\begin{array}{llll}\text { Región } & y & \text { Sociedad, } & \text { 15(26), }\end{array}$ http://www.scielo.org.mx/pdf/regsoc/v15n26/v15n26a2.pdf

Federación de Psicólogos de la República Argentina (FePRA) (2013). Código de Ética de la Fe.P.R.A. http://fepra.org.ar/docs/C_ETICA.pdf

Hu, L. T., \& Bentler, P. M. (1999). Cutoff criteria for fit indexes in covariance structure analysis: Conventional criteria versus new alternatives. Structural Equation Modeling: A Multidisciplinary Journal, 6(1), 1-55. https://doi.org/10.1080/10705519909540118

Kelley, K. (2007). Methods for the Behavioral, Educational, and Social Sciences: An R Package. Behavior Research Methods, 39, 979-984. https://doi.org/10.3758/BF03192993

Ley $\mathrm{N}^{\mathrm{o}}$ 25.326. Protección de datos personales. http://infoleg.mecon.gov.ar/ 
Linzalone, N., Coi, A., Lauriola, P., Luise, D., Pedone, A., Romizi, R., ... \& Maurello, M. T. (2017). Participatory health impact assessment used to support decision-making in waste management planning: A replicable experience from Italy. Waste management, 59, 557-566. https://doi.org/10.1016/j.wasman.2016.09.035

Lloret, S., Ferreres, A., Hernández, A., \& Tomás, I. (2017). The exploratory factor analysis of items: guided analysis based on empirical data and software. Anales de psicología, 33(2), 417-432. https://doi.org/10.6018/analesps.33.2.270211

López, J. S., Blanco. F., Scandroglio, B., \& Rasskin Gutman, I. (2010) Una aproximación a las prácticas cualitativas en psicología desde una perspectiva integradora. Papeles del Psicólogo, 31(1), 131-142. http://www.papelesdelpsicologo.es/pdf/1803.pdf

Marcon, A., Nguyen, G., Rava, M., Braggion, M., Grassi, M., \& Zanolin, M. E. (2015). A score for measuring health risk perception in environmental surveys. Science of The Total Environment, 527-528,

270-278. https://doi.org/https://doi.org/10.1016/j.scitotenv.2015.04.110

Martin, W. E., Martin, I. M., \& Kent, B. (2009). The role of risk perceptions in the risk mitigation process: The case of wildfire in high risk communities. Journal of Environmental Management, $\quad$ 91(2), 489-498. https://doi.org/http://dx.doi.org/10.1016/j.jenvman.2009.09.007

Martínez de Rusconi, M. \& Maffrand, G. (2014). Consideraciones de los barrios ciudades desde el enfoque urbano-ambiental. http://blog.ucc.edu.ar/ssh/files/2014/10/BARRIOS-CIUDADES-ENFOQUEURBANO-AMBIENTAL.pdf

Martínez Soto, J. (2004). Tesis de licenciatura no publicada. Facultad de Estudios Superiores Zaragoza, Universidad Autónoma de México. México.

Mayer, A., Shelley. T. O., Chiricos, T., \& Gertz, M. (2017). Environmental risk exposure, risk perception, political ideology and support for climate policy. Sociologica Focus, 50(4), 309-328. https://doi.org/10.1080/00380237.2017.1312855

McDaniels, T., Axelrod, L. J., \& Slovic, P. (1995). Characterizing perception of ecological risk. Risk Analysis, 15(5), 575-588. https://doi.org/10.1111/j.15396924.1995.tb00754.x

Mikulic, I. M., Cassullo, G. L.. Albornoz, O., Marconi, A., Fernandez, G., Ojeda, G. M., \& Torcassi, V. (2009). Psicología Ambiental y Evaluación Psicológica: Aportes de las técnicas psicométricas a la evaluación de la percepción del riesgo. I Congreso Internacional de Investigación y Práctica Profesional en Psicología XVI Jornadas de Investigación Quinto Encuentro de Investigadores en Psicología del MERCOSUR. Facultad de Psicología, Universidad de Buenos Aires. https://www.aacademica.org/000-020/762.pdf

Mikulic, I. M., Cassullo, G. L., Crespi, M. C., Caruso, A. P., Elmasian, M., \& Muiños, R. (2012). Evaluación de la percepción de riesgo en diferentes grupos sociales: propuesta de un modelo de ecuaciones estructurales. Anuario de investigación de la Facultad de Psicología. UBA, 19(2), 37-44. https://www.redalyc.org/articulo.oa?id=369139948042

Mikulic, I. M., Cassullo, G., Fernández, G., Giardina, E., Paolo, A., Caballero, R., \& Aruanno, Y. (2012). Estudio de la valoración de las situaciones de riesgo en estudiantes universitarios desde la perspectiva de la psicología ambiental. Trabajo presentado en IV Congreso Internacional de Investigación y Práctica Profesional en Psicología, Facultad de Psicología, Universidad de Buenos Aires, Buenos Aires. https://www.aacademica.org/000-072/49.pdf

Muthén, L. K., \& Muthén, B. O. (1998-2011). Mplus User's Guide (6th ed.). Los Angeles, CA: Muthén \& Muthén. 
Ohe, M., \& Ikeda, S. (2005). Global warming: risk perception and risk-mitigating behavior in Japan. Mitigation and Adaptation Strategies for Global Change, 10(2), 221-236. https://doi.org/10.1007/s11027-005-6138-6

R Core Team (2018). R: A language and environment for statistical computing. R Foundation for Statistical Computing, Vienna, Austria. https://www.R-project.org

Red Ciudadana Nuestra Córdoba (2014). Indicadores ciudadanos Nuestra Córdoba 2014. http://www.nuestracordoba.org.ar/sites/default/files/Indicadores_Ciudadanos_2014_0. pdf

Rodriguez-Sanchez, C., \& Sarabia-Sanchez, F. J. (2017). Perceived risk of urban water consumption: Scale development, validation and characterisation in Spain. Urban Water Journal, 14(4), 354-360. https://doi.org/10.1080/1573062X.2016.1148179

Rohrmann, B. (2008). Risk perception, risk attitude, risk communication, risk management: A conceptual appraisal. The International Emergency Management Society (Ed.), Global co-coperation in emergency and disaster management - 15th TIEMS Conference booklet.

http://tiems.info/dmdocuments/events/TIEMS_2008_Bernd_Rohrmann_Keynote.pdf

Rufat, S. (2015). Towards a social and spatial risk perception framework. Cybergeo: European Journal of Geography. https://doi.org/10.4000/cybergeo.27010

The World Meteorological Organization (WMO), UN Environment (UNEP), Intergovernmental Panel on Climate Change (IPCC), Global Carbon Project, Future Earth, Earth League and the Global Framework for Climate Services (GFCS) (2019). United in science. Documento producido para la Cumbre sobre la Acción Climática de las Naciones Unidas. https://library.wmo.int/index.php?lvl=notice_display\&id=21523\#.YRF40IhKjIV

Sjöberg, L. (1996). A discussion of the limitations of the psychometric and cultural theory approaches to risk perception. Radiation Protection Dosimetry, 68(3-4), 219-225. https://doi.org/10.1093/oxfordjournals.rpd.a031868

Slovic, S., \& Slovic, P. (2015). Numbers and nerves: Information, emotion, and meaning in a world of data. Oregon: Oregon State University Press.

Slovic, P., \& Weber, U. E. (2002, April). Perception of risk posed by extreme events. Conference Risk Management Strategies in an Uncertain World, Palisades, New York. https://www0.gsb.columbia.edu/mygsb/faculty/research/pubfiles/5461/perception\%20 of\%20risk.pdf

Stanojlovic, M. (2015). Percepción social de riesgo: una mirada general y aplicación a la comunicación de salud. Revista de Comunicación y Salud, 5(1), 99-110. https://doi.org/10.35669/revistadecomunicacionysalud.2015.5(1).99-110

Tabachnick, B. G., \& Fidell, L. S. (2013). Using multivariate statistics. Boston: Allyn and Bacon.

Weber, E.U. (2001). Risk: Empirical studies on decision and choice. In N. J. Smelser, \& P. B. Baltes (Eds.), International Encyclopedia of the Social \& Behavioral Sciences (pp. 13347-13351). Oxford: Elsevier.

Weber, J. M., Hair, J. F., \& Fowler, C. R. (2000). Developing a measure of perceived environmental risk. The Journal of Environmental Education, 32(1), 28-35. https://doi.org/10.1080/00958960009598669 